\title{
Chemomechanically voxelated niches for programmable histogenesis
}

Peter L. H. Newman*1, Pierre Ostei1 ${ }^{2,3}$, Tim A. Anderson ${ }^{1}$, Jane Q. J. Sun ${ }^{2,3}$, Daryan Kempe ${ }^{4}$, Maté Biro $^{4}$, Jae-Won Shin ${ }^{5}$, Patrick P.L. Tam ${ }^{2,3}$, Hala Zreiqat*1

${ }^{1}$ ARC Training Centre for Innovative Bioengineering, The University of Sydney, Sydney, Australia.

${ }^{2}$ Embryology Unit, Children's Medical Research Institute, Sydney, Australia

${ }^{3}$ School of Medical Science, Faculty of Medicine and Health, The University of Sydney

${ }^{4}$ EMBL Australia, Single Molecule Science Node, School of Medical Sciences, UNSW, Sydney, Australia.

${ }^{5}$ Department of Pharmacology and Regenerative Medicine; Department of Bioengineering, University of Illinois at

Chicago, Illinois, USA.

*Corresponding authors, Peter Newman: p.newman@sydney.edu.au, Hala Zreiqat: hala.zreiqat@sydney.edu.au

Tissue and organoid models have been established with increasingly physiological shape, size, and function ${ }^{1-3}$. However, histogenesis proceeds stochastically within these models, relying on 'self-organization' mechanisms that limit their ability to form recapitulative organotypic structures with controlled architecture and composition. To address this, we develop a printing technology to program histogenesis using material-guided instructive cues. We print voxelated niche microenvironments with independently tunable chemical and mechanical microproperties, or 'chemomechanics'. This includes the voxelization of conjugated peptides, proteins, and morphogens across a range of Young's Moduli. We show that these niches are capable of the cellular-scale programming of functions that underpin histogenesis, including mechanosensing and the differentiation of selective cell types. By rationally designing these niches with specific voxelated properties, we can program histogenesis and generate spatially reproducible tissues from a single cell-source. These tissues include a bone-fat-osteoid from stromal mesenchyme and a polarized assembly of germ-layer tissues derived from pluripotent stem cells. While programming germ-layer tissues, we reveal that polarized matrix mechanics can direct human germ-layer histogenesis in a model of tissue development. Thus, chemomechanically voxelated niches are a valuable tool to program and structure cellular-scale behaviors into well-defined tissues. Their continued study potentiates a better understanding of how extrinsic niche factors regulate histogenesis, and their application enhances capabilities for generating tissues and organ systems with a well-defined composition and architecture.

Generating tissue ex vivo with biologically relevant architecture and physiologically compatible functions remains a key challenge of tissue engineering. While there has been significant progress generating tissue and organoid models of increasing complexity ${ }^{1-6}$, these approaches use homogeneous extracellular environments, limited to stochastically self-organized tissues absent of 
the requisite structure for the emergence of higher-level cellular functions. One method to overcome this limitation is to support cells with complex heterogeneous niches with well-defined chemical and mechanical microproperties able to program local cell interactions and assemble these into tissue-typic and organotypic entities. To this end, researchers have developed biomaterials with properties that mimic the mechanics ${ }^{7-9}$ and chemistry ${ }^{10-14}$ of the cell niche. However, there are no existing technologies to generate synthetic niches that support multilineage tissue constructs with reproducible structure.

One promising technology to generate complex niche materials is 3D-printing. One standard printing method involves the extrusion of a biopolymer ink or the direct extrusion of cells. Extrusion printers can produce materials with discrete properties through the sequential extrusion of different materials, either from separate print cartridges, each loaded with different bioinks ${ }^{15-19}$, or through mixing solutions before their extrusion through a single nozzle ${ }^{20,21}$. Using this approach, researchers have demonstrated the structuring of differing cell types ${ }^{3,18}$ mechanical properties ${ }^{17,21}$ and biochemicals ${ }^{22}$. Structured materials fabricated with extrusion printing are limited in the number of different material types by the number of print cartridges or their ability to switch and mix solutions within a single print cartridge efficiently. Accordingly, structured cell systems fabricated from extrusion printing have been limited to no more than three cell inks ${ }^{19,23}$, nor been demonstrated with comparable feature sizes to those possible through photolithographic methods ${ }^{24}$. These limitations make the extrusion approach problematic for fabricating niche materials with microscale voxelization. As an alternative approach to indirectly structuring tissues with niche cues, organoid models have been generated using direct extrusion printing of hiPSCs ${ }^{3,25,26}$. IPSCs can be directly extruded into defined architectures and subsequently differentiated via external 60 media-derived cues or intercellular feedback from concurrently patterned differentiated cells. While this approach is permissive to generating macroscale organoids with improved reproducibility, these models still rely on the stochastic self-organization processes limiting their size and preventing the emergence of higher-level function.

Photolithography is an alternative printing method that uses light to selectively polymerize a material from a photoresist. This method offers technical solutions to some of the obstacles faced when extrusion-printing complex materials. For example, photolithographic printing methods have generated materials with nanoscale features of $30 \mathrm{~nm}^{27}$, a feat yet to be achieved using extrusionprinting. Early work developing heterogeneous biomaterials with chemically discrete properties used photolithography to pattern regions of small bioactive molecules and peptides ${ }^{28-30}$. However,

70 since then, methods for the discrete spatiotemporal structuring of sensitive and complex biomacromolecules have been developed ${ }^{31}$, as well as methods for printing materials with discrete 
mechanical properties ${ }^{32}$. Photolithographic methods print materials with heterogeneous properties by changing the composition of a photoresist during printing. In practice, this is done by serial injection of photoresists with different composition $^{33}$. These photoresists flow through the polymerization volume, which gives this method the name flow lithography (FL). The FL method has been widely used in microfluidics to fabricate microparticles ${ }^{34}$. FL overcomes the technical hurdles of extrusion methods by using separate subsystems for solution injection, mixing, and placement/polymerization. Thus, for FL printing, materials with structured properties can be fabricated with a resolution primarily limited by the focus of light, the so-called 'spot-size. While previous works using photolithography have generated niches that regulate cell attachment ${ }^{24,30}$ and proliferation $^{31}$, the spatial regulation of mechanosensing, cell differentiation, and their assembly into multilineage constructs remain unexplored.

Here we present a printing technology to program histogenesis using chemomechanically voxelated niches. We demonstrate cellular-scale programming of mechanosensing and the differentiation of selective cell types. Niches with specific voxelated properties, can program histogenesis and generate spatially reproducible tissues from a single cell-source, including a bonefat-osteoid and a niche-polarized germ-layer tissue. While programming germ-layers, we reveal that matrix mechanics can direct human germ-layer histogenesis in vitro.

\section{Results.}

90 Printing of voxelated niches using chemomechanical flow lithography. Niches were fabricated by flowing photoresists of variable biochemical and polymeric composition through a print chamber during printing (Fig. 1, Fig. S1,2). Changing the laser's focus controls the resolution and size of printed structures, with a minimal resolution demonstrated to $7 \mu \mathrm{m}$ (Fig. 1b-1, Fig. S3). The photoresist is composed of an inert hydrogel monomer (polyethylene glycol diacrylate), a 95 photoinitiator (lithium phenyl-2,4,6-trimethylbenzoylphosphinate or LAP ${ }^{35}$ ), and biochemicals, which can include any peptide, protein, or morphogen with a thiol-functional-group available from cysteine peptide moieties (Fig. 1c). Following laser-induced photocleavage of the photoinitiator, the hydrogel monomer polymerizes alongside a thiol-ene bioconjugation reaction that can covalently crosslink biochemicals to the otherwise bioinert niches. Thus, the inclusion of

100 biochemical additives specifies niche bioactivity (Fig. 1b-5,6, Fig. S4). Likewise, physiologically stiff (Fig. 1b-4 Fig. 1f) and soft (Fig. 1b-5, Fig. 1g) Young's moduli can be printed by changing the monomer and photoinitiator concentration. Coordinating the above, CMFL can print 3D architectures that support cell attachment and growth (Fig. 1 h, i). 


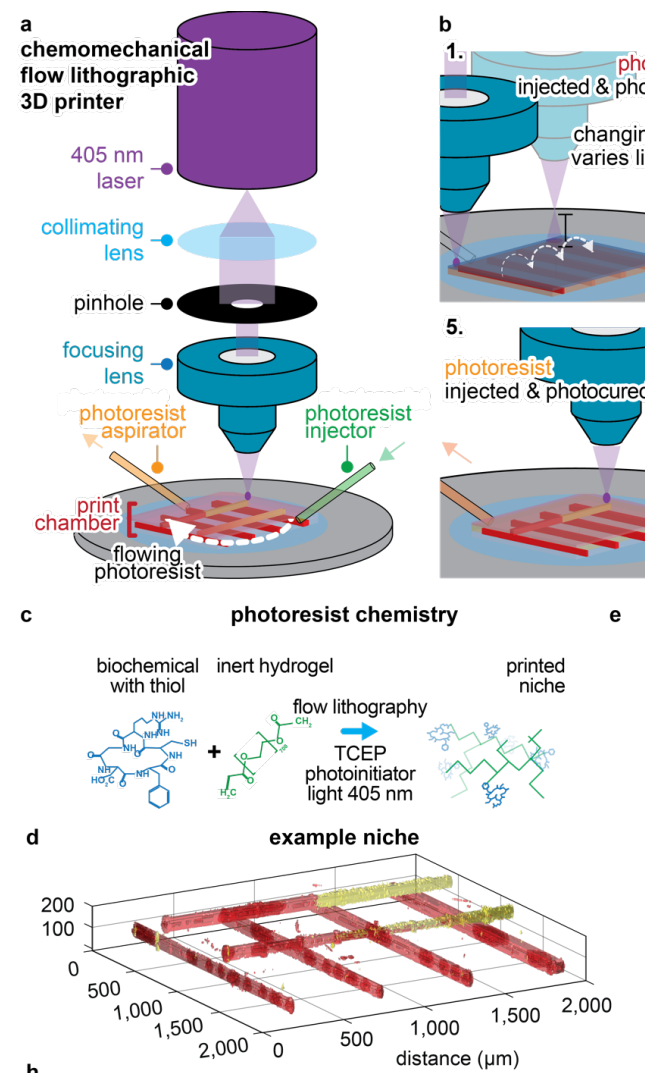

h

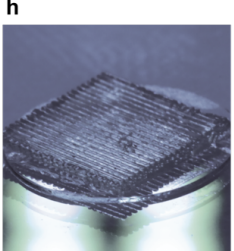

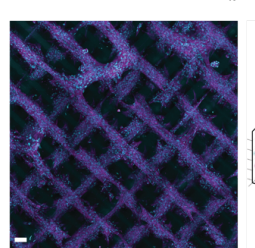

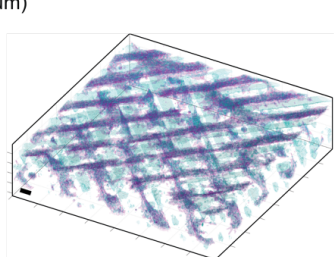

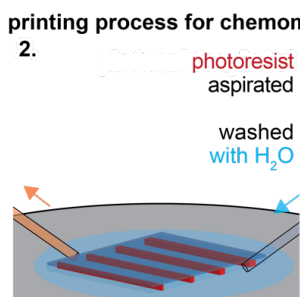

6.
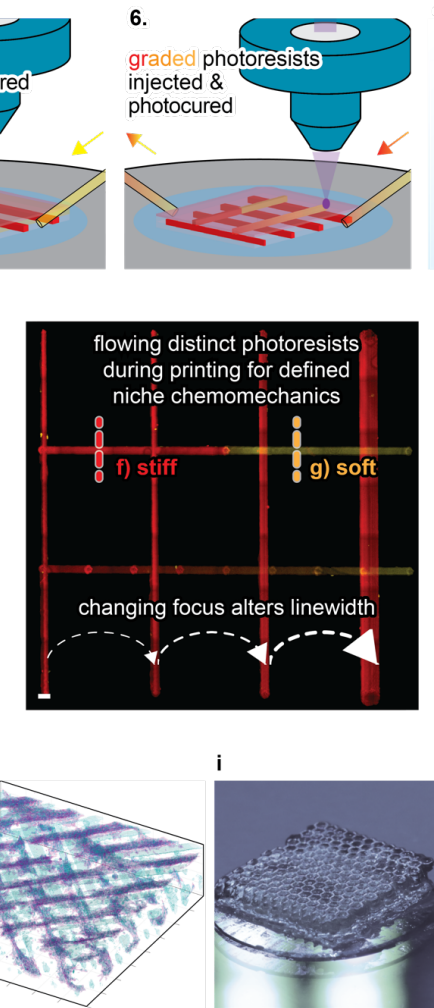

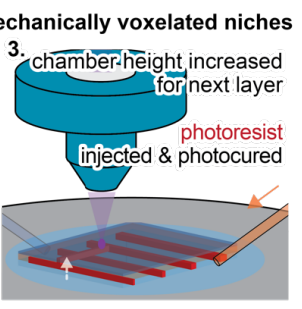

7.

cells seeded onto heterogeneous microproperty cues

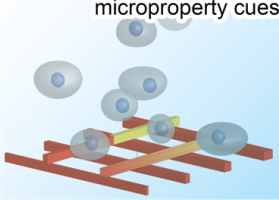

4.
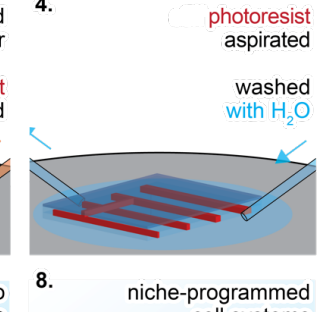
cell systems
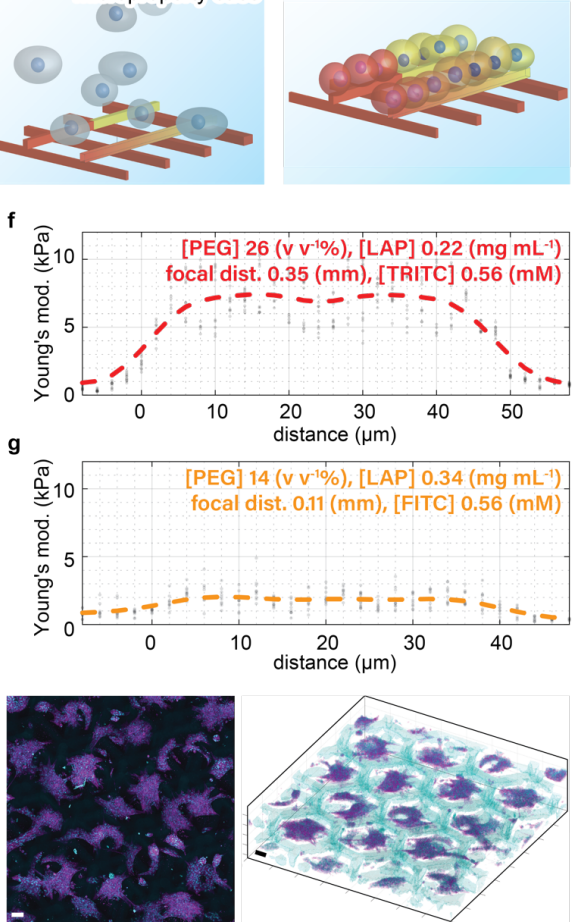

$1 \mid$ Chemomechanical flow lithographic printing of voxelated niches. a, Components of the CMFL 3D-printer. $\mathbf{b}$, Stepwise fabrication processes to generate niches. c, Photoresist chemistry used. d, Confocal image of an example niche with voxelated properties, including changes to linewidth, mechanics (Young's modulus), and chemical microproperties (concentration of the fluorophores FITC and TRITC). e, Maximum intensity projection of the 3D niche confocal data, scale bar $50 \mu \mathrm{m}$. Dotted red and orange lines annotate the profile of force spectroscopy in $\mathbf{f}, \mathbf{g}$. Young's modulus across filaments of the pictured niches. Fabrication variables are shown at the top of the graphs for physiologically $\mathbf{f}$ stiff ( $7.5 \mathrm{kPa}$, red, TRITC) and $\mathbf{g}$ soft ( $2.5 \mathrm{kPa}$, orange, FITC) segments. Actin (magenta) and hydrogel (cyan) stained ADSCs (primary human adipose-derived stromal cells) cultured over 3D niche scaffolds with $\mathbf{h}$, 'stacked-logs' or $\mathbf{i}$, 'offset-honeycomb-layers' architecture. Macro lens photography is show beside maximum intensity projections and 3D confocal renders, scale bars $200 \mu \mathrm{m}$.

To print independently tunable niche chemomechanics, we developed a model that relates the

115 CMFL fabrication variables to the printed linewidth, Young's Modulus, and conjugated thiol-ene biochemical of the printed hydrogels. We characterized six variables affecting niche properties, including three variables controlled by the printer (laser scan velocity, focus, and laser power) and three photoresist variables (the concentration of photoinitiator, monomer, and bioconjugate BiotinPEG-SH, a model biochemical with free thiol group) (Fig. 2 a-f). Decreased light exposure (Fig.

120 2a,b), photoinitiator (Fig. 2d), and monomer concentrations (Fig. 2e) decreased both Young's modulus and the linewidth of prints, consistent with a lowered rate of polymerization due to the reduced light absorption and consequentially lower photoinitiator cleavage and monomer 
conversion (see notes on photopolymerization in the supplementary information, Fig. S5-6). The diameter of the conic angle of laser transmittance linearly correlated to changes in linewidth (Fig.

125 2c). Additionally, we characterized the effect of changing Biotin-PEG-SH concentration (a model biochemical with a free thiol group) on niche Young's modulus and linewidth. Here, Biotin-PEGSH inclusions up to $8 \mathrm{mM}$ did not significantly alter Young's modulus or linewidth (Fig. 2f).

A variable state-space that simplifies the number of independent printing variables while still exhibiting physiologically relevant niche properties was characterized (Fig. 2g-i). This 130 simplification was achieved by fixing the laser scan velocity to $100 \mathrm{~mm} \mathrm{~min}^{-1}$ and laser power to $100 \%$, as well as unified the two variables of photoinitiator and monomer concentration to a single variable by changing the concentration of one as a function of the other (Methods, Equation 2). These simplifications left a state-space with the three independent variables of focus, bioconjugate concentration, and the combined concentration of photoinitiator-monomer. Niches with voxelated

135 microproperties could then be printed by interpolating the relevant fabrication variables from the simplified state-space (Fig. 2g-i, see methods for additional details). We demonstrated the power of this approach by printing adjacent niches filament structures with permutations of either increasing or decreasing relative Young's Moduli, linewidth, and relative bioconjugate fluorescence (Fig. S7). This approach allows specification of chemomechanically voxelated niches

140 with Young's Moduli between 2-20 kPa, biochemical additives to $4 \mathrm{mM}$, and with linewidths from 40 to $300 \mu \mathrm{m}$ (Fig. 2 g-i). 

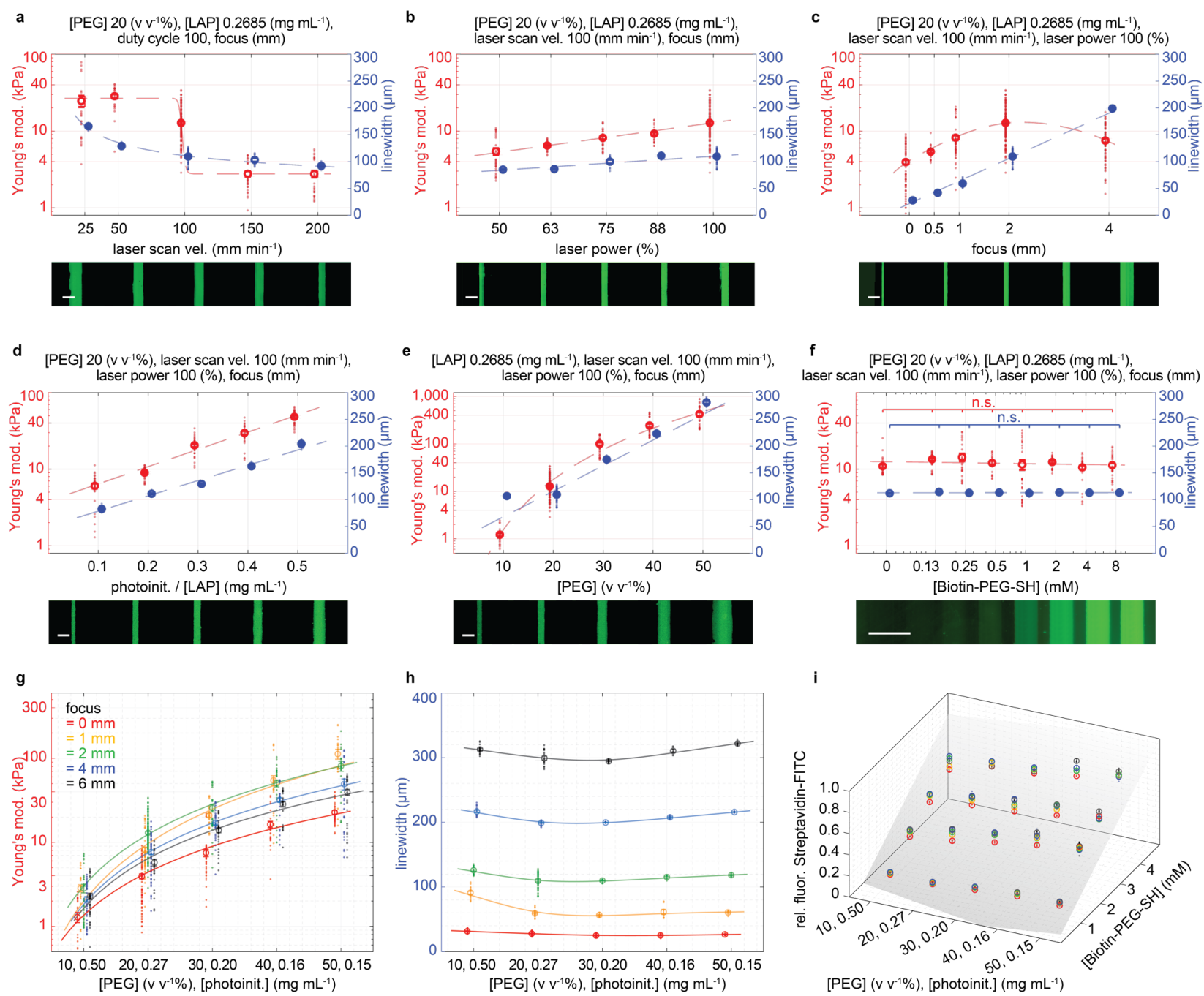

Fig. 2 | Printing model for voxelated niche chemomechanics. a-f The effect of CMFL device and photoresist variables on niche Young's modulus (left axis, red) and linewidth (right axis, blue). For each variable subset, an image of the printed niche with streptavidin-FITC conjugate is shown. Gamma correction is applied to subset $\mathbf{f}$ for improved visibility. Fabrication variables that remain constant are enumerated at the top of each subset. Scale bars, $200 \mu \mathrm{m}$. g-i, The optimized variable state-space. CMFL niches with well-defined properties can be interpolated from the optimized state-space to generate niches with complex microstructure. $\mathrm{g}$, The relationship between the unified photoinitiator-monomer concentration (horizontal axis) and focus (shown in different colors) on Young's modulus and $\mathbf{h}$, linewidth. i, The effect of photoinitiator-monomer (horizontal axis), Biotin-PEG-SH concentration (intopage), and focus (shown in different colors) on the bound thiol-ene conjugate. n.s. $-p>0.05$ by one-way ANOVA with Bonferroni post hoc tests. Mean \pm s.e.m. Throughout the figure, data points and fit lines are offset on the horizontal-axis to minimize overlap of concurrently shown dependent variables.

Niche-programmed cell attachment, spreading, and mechanosensing. We explored if voxelated niches could spatially program changes to cellular-scale functions. To test this, we printed niches with parallel filaments between 50-250 $\mu \mathrm{m}$, with physiologically moderate Young's modulus of $8 \mathrm{kPa}^{36}$ and alternating concentrations of the cell attachment peptide RGD. (cyclo(ArgGly-Asp-d-Phe-Cys)) (Fig. 3a, Table S1). We then cultured multipotent primary human adiposederived stromal cells (hADSCs) for $72 \mathrm{~h}$ on niches. Cells selectively attached to regions printed with the RGD peptide conjugate, increasing cell spreading over structures with larger linewidths. 
160 We then explored if niches with voxelated RGD microproperties could regulate cell attachment and spreading in a dose-dependent fashion. Accordingly, we printed niches with six voxelated regions of RGD concentrations between $0-8 \mathrm{mM}$, a moderate Young's modulus ( $8 \mathrm{kPa})$, and a fixed linewidth $(250 \mu \mathrm{m})$ (Fig. S10, Table S1). Here we observed cell attachment and spreading reflected the underlying niche RGD concentration, demonstrating that the niche chemistry can spatially define microscale changes to cell attachment and spreading.

This result led us to postulate that voxelated niches could spatially program more complex cell functions, including cellular-scale changes to cell mechanosensing. To test this, we studied the cellular mechanostat YAP/TAZ, a widely explored marker for determining cellular mechanosensing within multipotent stromal cells ${ }^{14,37-39}$. As previously used, we printed niches with

170 voxelated RGD, as well as niches with voxelated Young's Moduli between 2.5-20 kPa, ranging from physiologically soft-to-stiff (Fig. 3c, Table S1) ${ }^{36}$. We measured cell mechanosensing by quantifying the relative distribution of YAP/TAZ in the nucleus $(\mathrm{N})$ and the cytoplasm $(\mathrm{C})$ by assessing the $\mathrm{N}: \mathrm{C}$ ratio of average fluorescent intensity in these two intracellular compartments. The YAP N:C ratio revealed that microscale cell mechanosensing could be regulated through the 175 underlying chemical and mechanical (RGD, Fig. 3b, Young's Moduli, Fig. 3c) microproperties of niches $^{37-39}$. Therein cell mechanosensing exhibited a sigmoidal response to changes in the microstructured concentration of RGD and Young's modulus. A lower threshold of YAP N:C correlated with a low concentration of RGD and soft Young's modulus, while for high concentrations of RGD and stiff Young's modulus, an upper of YAP N:C was observed. Thus, we

180 show that voxelated niches can program microscale changes to cell mechanosensing, a finding with significant implications for generating tissue ex vivo, as YAP signaling plays an essential role during development as a critical juncture in Hippo pathway signaling of histogenesis, morphogenesis and organ growth ${ }^{40}$. 

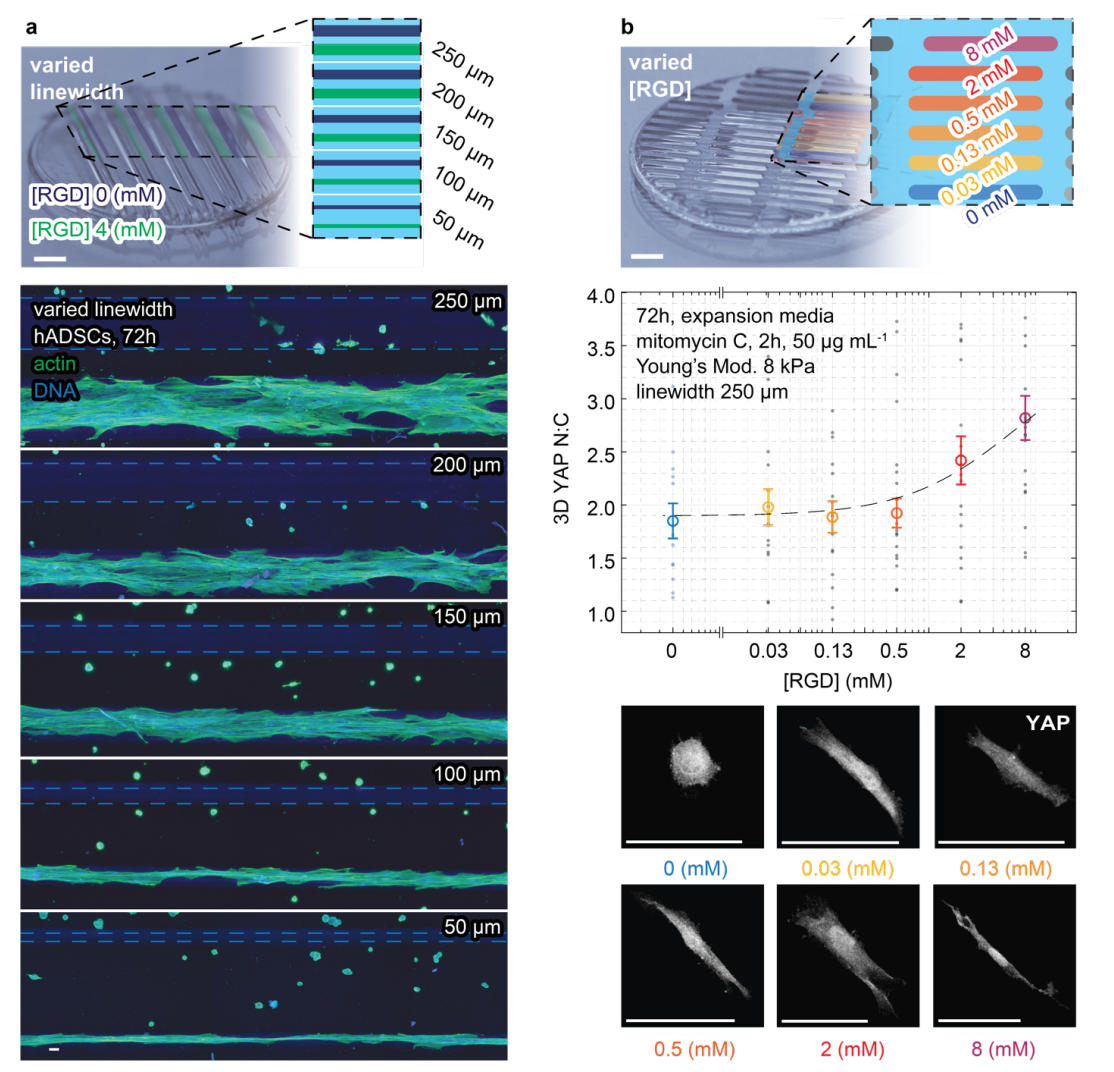

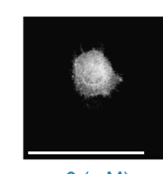

$0(\mathrm{mM})$

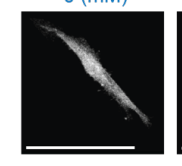

$0.5(\mathrm{mM})$
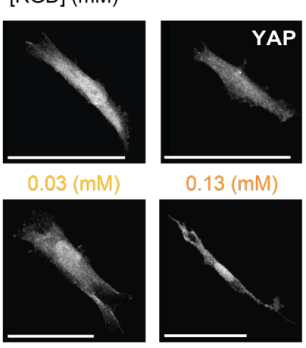

$2(\mathrm{mM})$

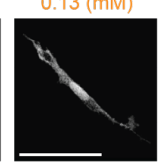

$8(\mathrm{mM})$
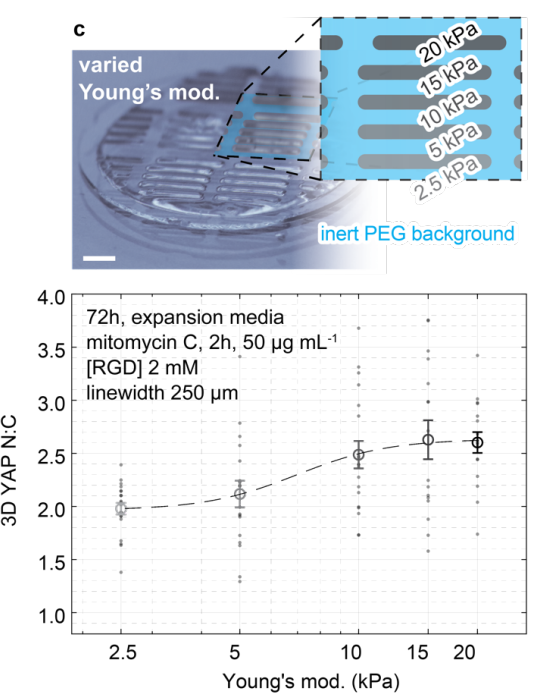

Young's mod. $(\mathrm{kPa})$

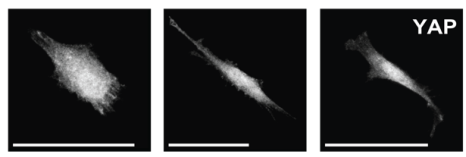

$2.5(\mathrm{kPa})$

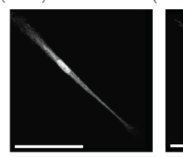

$15(\mathrm{kPa})$

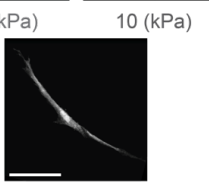

$20(\mathrm{kPa})$

185 Fig. 3 | Niche-programmed cell attachment, spreading, and mechanosensing. a, Printed line-pairs with 4 or 0 mM RGD, from $50-250 \mu \mathrm{m}$, with a fixed Young's modulus of $8 \mathrm{kPa}$. Representative confocal image shows actin (phalloidin, green) and nucleus (DAPI, blue). Gamma-correction has been applied to improve the visibility of the zero RGD concentration dark blue filaments where no cells attach. Niche arrays with voxelated $\mathbf{b}$, chemical (RGD) and $\mathbf{c}$, mechanical (Young's modulus) properties are shown alongside the quantification and representative MIP images of the 3D confocal nuclear (N) to cytoplasmic (C) ratio of biomolecular mechanostat YAP in hADSCs at $72 \mathrm{~h}$ as imaged on their respective microproperty regions. Mean \pm s.e.m. Scale bars, $50 \mu \mathrm{m}$, except macro lens images of CMFL arrays at figure top $=1 \mathrm{~mm}$.

\section{Niche-programmed histogenesis of a spatially reproducible bone-fat-osteoid from stromal}

mesenchyme. Given the upstream role of cellular mechanosensing in cell fate decisions ${ }^{37}$, we tested if cell differentiation could be spatially programmed from chemically (RGD peptide) and mechanically (Young's Modulus) voxelated niches. Harnessing the propensity of osteogenic and adipogenic differentiation of the multipotent stromal cells ${ }^{14,38,41}$, we sought to assemble a bone-fat structure resembling an osteon through optimized niche interactions. We assessed osteogenesis by measuring the nucleocytoplasmic $(\mathrm{N}: \mathrm{C})$ ratio of RUNX2, an essential transcriptional regulator of early osteogenesis coupled with the commitment of multipotent stromal cells to the osteoblast

200 lineage $^{42}$, and by visualizing mineralized bone deposition by Alizarin Red staining. Enhanced osteogenesis, revealed by elevated RUNX2 N:C ratio and enhanced mineralization, was correlated with increasing concentrations of RGD. (Fig. 4a,b) and stiffer Young's modulus (Fig. 4c,d). Adipogenesis, assessed as the volumetric ratio of fat-to-total-cytoplasmic-volume (Fig. 4e,f), was correlated with mechanically soft (Young's modulus) or peptide-enriched (high RGD) regions of 
205 the niches. The highest fat-cytoplasmic volumetric ratio was associated with mechanical microproperties with $8 \mathrm{mM}$ RGD and a low Young's modulus (with $\sim 7$-fold increase at $2.5 \mathrm{kPa}$ relative to $20 \mathrm{kPa}$ ). Further, we tested niches voxelated with varying concentrations of the morphogen BMP2, known to influence osteogenesis. Niches with varied BMP2 were assayed for osteogenesis, showing that RUNX2 N:C scaled up with BMP2 concentration up to $200 \mathrm{ng} \mathrm{mL}{ }^{-1}$,

210 but decreased at $1000 \mathrm{ng} \mathrm{mL} \mathrm{m}^{-1}$. For Alizarin Red staining, mineralization was shown to increase across all ranges of BMP2, suggesting that high concentrations of BMP2 may have accelerated osteogenesis, passing peak RUNX2 expression at 72 h (Fig. 4g,h).

We next sought to assemble multilineage cell differentiation from a single cell source to generate an osteon-like tissue, or 'osteoid' with a well-defined structure. To elicit concurrent cell 215 differentiation of human adipose-derived stem cells (hADSCs) to both osteo-and-adipogenic lineages, we printed niche arrays with voxelization that reflects the optimal niche microproperties previously explored for osteo-and-adipogenic differentiation (Fig 4. a-h). Therein a centralized adipogenic region is surrounded peripherally by an osteogenic region (Fig. 4i). hADSCs were seeded over the bone-fat voxelated niche and cultured in a 1:1 adipogenic:osteogenic media for 14 220 days, displayed concurrent osteogenesis (COL 1A-expressing, Fig. 4j) and adipogenesis (high lipid content, Fig. 4j) in their respective spatial domains (Fig. 4k, Fig. S11). Adipogenesis was localized to the center of the niche, over the region of low stiffness and high RGD, with enhanced osteogenesis over the niche periphery with high stiffness, BMP2, and RGD. This experiment showed that rationally designed voxelated niches can program local cell differentiation to generate tissue-like multilineage entities with a controlled structure. 

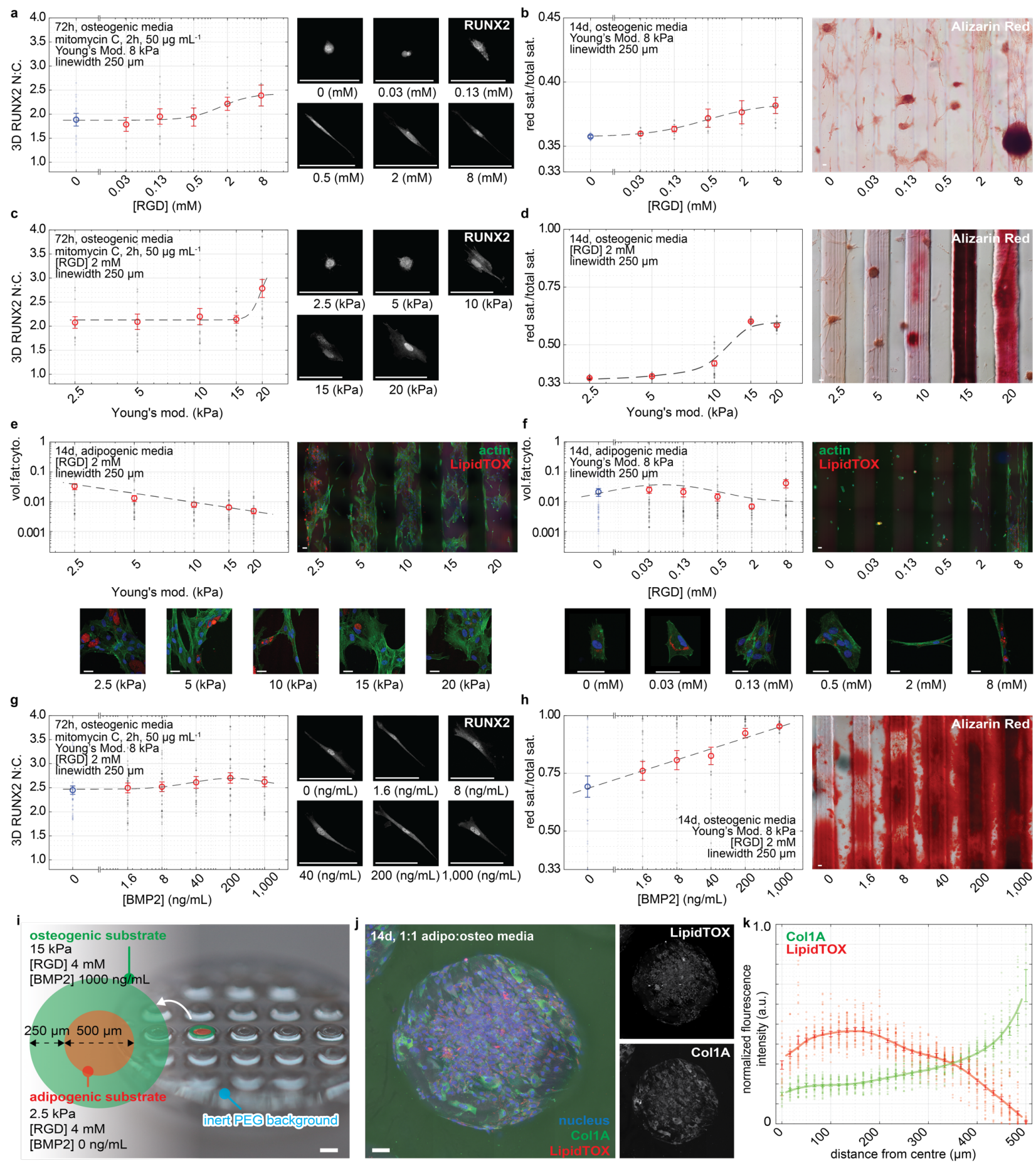

Fig 4 | Niche-programmed histogenesis of a bone-fat-osteoid from stromal mesenchyme. a,b, Osteogenic differentiation of hADSCs on chemically (RGD) microstructured niches. a, Immunofluorescent staining, imaging and quantification of RUNX2 and $\mathbf{b}$, quantification and representative widefield images of Alizarin Red stained bone mineralization. c,d, Osteogenic differentiation on mechanically (Young's modulus) microstructured niches, with RUNX2 and Alizarin Red stains. e,f Adipogenic differentiation over e, mechanically (Young's modulus) and f, chemically (RGD) microstructured niches, with representative max intensity projections and quantification of LipidTOX stained fat volume per cytoplasmic volume (vol. fat:cyto). g,h, Osteogenic differentiation on microstructured concentrations of the bioconjugated morphogen and growth factor BMP2, with RUNX2 and Alizarin Red stains. i-k, Organotypic bone-fat microstructures mimic the osteon's architecture with a central adipogenic region and a peripheral osteogenic region. i, Printed CMFL bone-fat niche array with optimized microproperties enumerated $\mathbf{j}$, Representative max intensity projections of CNA35 fluorescent collagen 1A (Col1A) probe and LipidTOX stained hADSCs. The large inset shows nuclear (blue), brightfield (grey), Col1 A (green), and LipidTOX (red) channels, beside smaller grayscale insets showing Col1A and LipidTOX channels, gamma-corrected for ease of visibility. $\mathbf{k}$, quantification of the normalized pixel intensity for Col1A and 
LipidTOX data across replicates. Scale bars $100 \mu \mathrm{m}$, except $\mathrm{d}=1 \mathrm{~mm}$. Data presented as mean \pm s.e.m. throughout. Scale bars 100 $\mu \mathrm{m}$, except for $\mathrm{i}=1 \mathrm{~mm}$.

\section{Niche-programmed mechanics recapitulate embryonic matrix softening and support} polarized germ-layer histogenesis. We next sought to broaden the application of CMFL and voxelated niches by demonstrating the spatially controlled histogenesis of a pluripotent stem cell derived germ-layer tissue ${ }^{1,43-46}$. To highlight the novelty and utility of these niches, we focused our research on studying a phenomenon that would otherwise be impossible to explore using alternative tools. We choose to research the role of matrix softening known to drive germ-layer formation and regionalization of tissues in the avian and murine embryos ${ }^{47,48}$. Using voxelated niches, we investigated if a similar process may exist in humans. First, we replicated the pattering of germ layer derivatives in tissue micropatterns in vitro, with the culture of hiPSCs plated on 1000 $\mu \mathrm{m}$ circular adhesive templates ${ }^{46,47}$. As per this predicate work, cells cultured on a homogenous template generated radially symmetric germ-layer derivatives (Fig. S14). We next modified this method by introducing a 'matrix-softened' region within a voxelated square niche. Such matrixsoftened mimetic niches were fabricated with a 1D gradient in their mechanics (Fig. 5d) alongside a control niche with uniform niche microproperties (Fig. 5a). hiPSCs cultured on both niche types were induced to differentiate with BMP4 $(50 \mathrm{ng} / \mathrm{mL})$ into progenitor cells of the mesoderm (BRApositive), endoderm (SOX17-positive), and ectoderm (SOX2-positive) ${ }^{1}$. Image analysis pooling replicate-data (Fig. S12) revealed that hiPSCs cultured on uniform stiffness substrate displayed regionalization of germ layer tissues from their center-to-periphery (Fig. $\mathbf{5 c}, \mathbf{j}, \mathbf{i}, \mathbf{k})$, similar to that observed for circular micropatterns ${ }^{1}$ (Fig. S14). In contrast, cells cultured over matrix-softened

260 mimetic niches were able to reconfigure the tissue pattern. Expression of BRA- and SOX17positive cells were localized to niche regions of low stiffness and SOX2-positive cells to regions with high stiffness (Fig. 5f,h,l). This observation reveals a novel role for niche mechanics in cellfate choices, suggesting that spatial variations in niche mechanics may underpin germ-layer tissue patterning during embryonic development. These findings are consistent with the notion that 265 protease softening of the basement membrane material plays a role in the patterning of tissues in the embryo's anterior-posterior axis described in murine and avian embryos ${ }^{47,48}$. Thus, CMFL and voxelated niches provide an entry point to better understand the mechanisms underpinning histogenic patterning processes in stem cell-based embryoid models. 


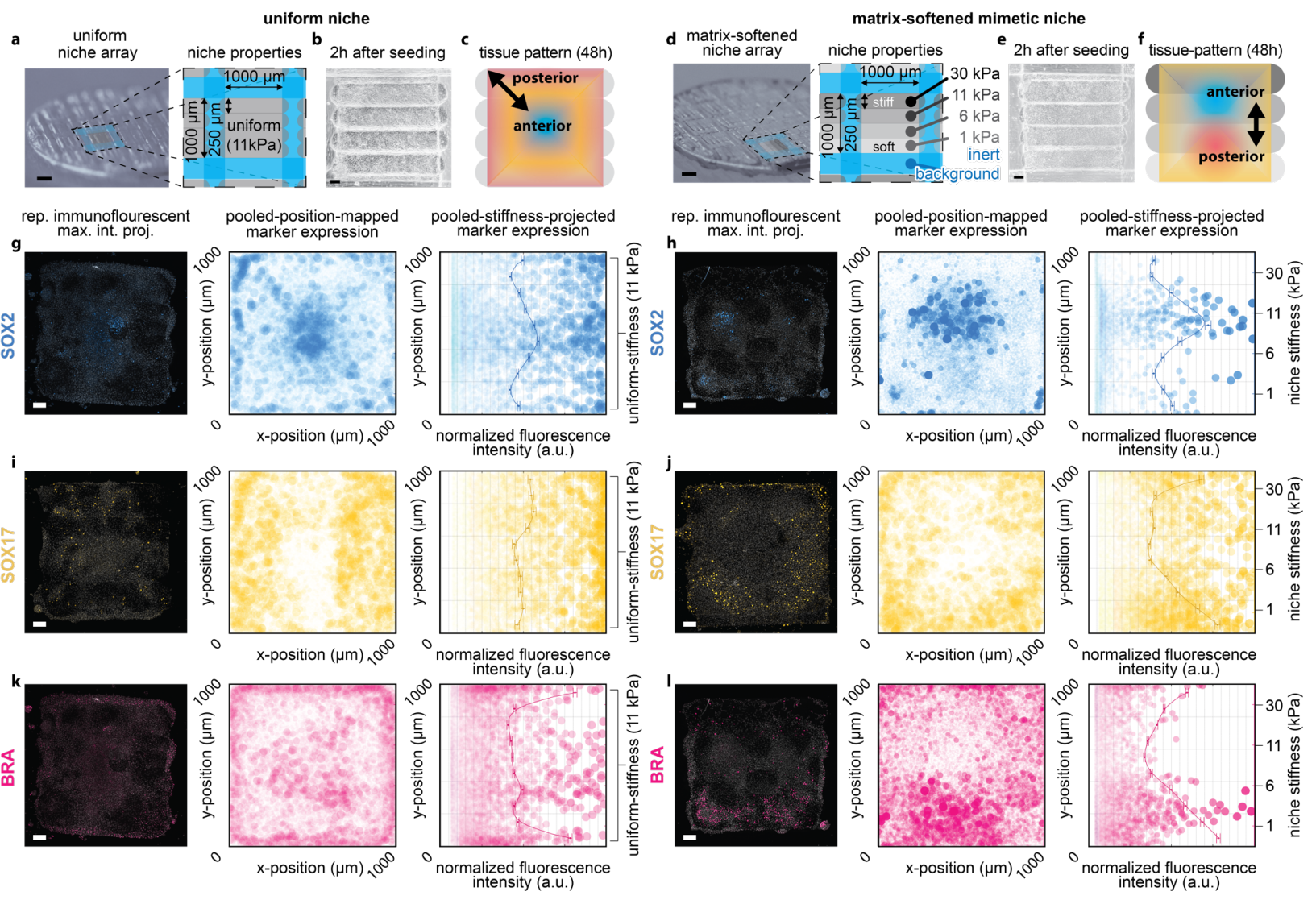

$270 \quad$ Fig. 5 | Niche-programmed mechanics recapitulate embryonic matrix softening and support polarized germ-layer histogenesis. a,d, Coverslip with niche arrays showing voxelization of niche microproperties. b,e, Brightfield imaging at $2 \mathrm{~h}$ following attachment. c,f, schematic summarizing germ-layer tissue-patterning of embryoid cultures 48h post-BMP4-treatment, showing (ectoderm: SOX2, blue and endoderm: SOX17, yellow; mesoderm: BRA, red). Representative immunofluorescent imaging of tissue cultures on niches with $\mathbf{g}, \mathbf{j}, \mathbf{k}$, uniform-stiffness, or $\mathbf{h}, \mathbf{j}, \mathbf{l}$, matrix-softened mimetic stiffness. Position-mapped expression graphs of replicate pooled-data show each marker to the right of immunofluorescent imaging. Each dot represents a single cell with size and transparency linearly correlated to the marker's normalized fluorescent intensity. The immunostained data in subsets top g$\mathbf{I}$ is gamma-corrected to improve visibility. Scale bars $100 \mu \mathrm{m}$, except a,d $=1 \mathrm{~mm}$.

Discussion. Chemomechanically voxelated niches offer the ability to program microscale changes to cell functions through independently definable niche interactions. While both 2D and 3D multi-

280 layer hydrogels can be fabricated using the CMFL method (Fig. 1), we validated the technology capacity of niche-programmed cell functions using single-layer (2D) hydrogels. We showed that voxelated niche properties can elicit cell mechanosensing, drive differentiation of stem/progenitor cell tissue, and program histogenesis to generate spatially reproducible multilineage tissue models. Future work using such niches may extend to multi-layer structures with increased complexity. In 285 such work, the fabrication of multi-layer niches could be achieved using chemical photoabsorbers for the facile photolithographic printing of 3D structures ${ }^{\mathbf{5 0}}$ or using multiphoton lithography. In particular, as multiphoton photolithography can achieve subdiffraction-limited resolutions ${ }^{27}$, combining multiphoton methods with FL may enable printing materials with voxelated niche nano properties. 
290 A further advancement to the CMFL method could be made by adopting a more specific conjugation chemistry. In particular, our method is limited when conjugating relatively complex macromolecules with numerous cysteine sites. Under these conditions, thiol-ene conjugation occurs stochastically and can lead to the bioconjugate's loss of function. This limitation has been addressed with more reproducible chemistries, including enzymatic methods ${ }^{31}$ and specific high

295 affinity non-covalent binding chemistries ${ }^{51}$. Further, such complex chemistries have been demonstrated with multiphoton photolithographic techniques ${ }^{24}$.

The use of chemomechanically voxelated niches complements recent research in pluripotent stem cell biology generating complex tissue and organoid models ${ }^{2,3}$. To date, these methods have relied on the self-organization of cells and tissues in relatively homogeneous niches ${ }^{52}$, with

300 microfluidically introduced morphogen patterning ${ }^{5}$, or the concurrent extrusion printing of different cell types ${ }^{3,18,23}$. Complex voxelated niches potentiates the next generation of reproducible synthetic tissue models, wherein histogenesis is defined through niche-mediated chemomechanical interactions. While it is feasible to reproducibly generate a bone-fat microtissue and polarized embryoid, the continued application of these niche systems has the potential to investigate some of 305 the biggest unanswered questions in biology, such as how complex structure and function emerge; how the shape, size, and body coordinates of an organism are determined; and how mechanical and positional morphogenetic cues works in concert with morphogens and lineage determinants. 


\section{Methods.}

Custom-built CMFL 3D-printer. The 3D-printer was built modifying a Hyrel System 30M. Custom components were added including, a $405 \mathrm{~nm} / 500 \mathrm{~mW}$ laser module and Arduino-based controller electronics, collimating and focusing lens (Thorlabs AL1225H and Olympus x10 Plan

315 NA 0.3 160/0.17), a pinhole/ring-actuated iris diaphragm (Thorlabs SM1D12D), and hydrophobic print chamber. All custom components of this system are detailed in the supplementary information.

CMFL niche print chamber. A photoresist is injected into a glass print chamber with a PDMS coated glass slide top, and acrylate functionalized coverslip base (Fig. S2). The acrylate-modified

320 glass coverslip base provides a stable surface on which microstructures are covalently attached when printed. Covalent binding between sample and coverslip prevents sample delamination, sample-folding, or strain deformation due to hydrogel swelling. Further, covalent attachment assists sample handling for cell culture and downstream characterization. To acrylate coverslips, a silanization solution was prepared. In a fumehood, a glass dish was filled with methanol (Sigma 322415) and bath sonicated for 5 minutes. The dish was then dried then rinsed a further $\mathrm{x} 2$ with methanol. $100 \mathrm{~mL}$ of methanol was added to the dish along with $5 \mathrm{~mL}$ of glacial acetic acid (Sigma A6283) and $3 \mathrm{~mL}$ 3-(trimethoxy silyl) propyl acrylate (TCI A1597). Circular glass coverslips were then washed $\mathrm{x} 3$ times in methanol in the glass dish, and a silanization solution was added before covering the dish to prevent evaporation or contamination from ambient H2O. Coverslips are left

330 for one hour for silanization to proceed. Following the reaction, coverslips were rinsed $\mathrm{x} 3$ in ethanol, wiped clean, and dried with $\mathrm{N}_{2}$. PDMS coating of glass slides was used at the top of the print chamber to prevent the bonding and tearing of photopolymerized niche samples. PDMS (Corning Sylgard 184) is prepared as ten parts base to one part curing agent, mixed, and then centrifuged to remove bubbles. Slides (Sigma CLS294775X50) are coated on Laurell EDC 650

335 series spin coater. PDMS is poured onto the top of the samples at approximately the volume of an Australian 50 cent piece. The spin coater is then ramped to $1600 \mathrm{rpm}$ for $10 \mathrm{~s}$ and stopped. The glass slides are then placed onto a hot plate at $200{ }^{\circ} \mathrm{C}$ for $1 \mathrm{~min}$ before being transferred to a $37{ }^{\circ} \mathrm{C}$ oven overnight.

Measurement of voxelated niche microproperties. Niches were fabricated on acrylated 340 coverslips using the geometries, photoresist, and printer variables as reported. The measurement of Young's modulus was completed via force spectroscopy using a JPK. NanoWizard Sense AFM mounted on Nikon Ti microscope. The device was fitted with the SuperCut quartz cantilever holder for liquid immersion and used with Bruker MLCT pyramidal cantilevers with stiffness calibrated using the thermal noise method. For force-displacement curve generation, hydrogel samples and 
345 AFM cantilever were submerged in x1 PBS. The cantilever approach velocity was fixed to $0.5 \mu \mathrm{m}$ $\mathrm{sec}^{-1}$ and terminated at a threshold force of $10 \mathrm{nN}$. Measurements were taken from 3 independent experimental replicates from at least four different printed-niche-replicates in each experiment. The Young's modulus was calculated from each force-displacement approach curve using a custom fitting program written in MATLAB, with automated contact point determination and fitting for an $18^{\circ}$ half-angle conic section (Sneddon model, as per Bruker recommendation for MLCT pyramidal cantilevers), with sample-thicknesses bottom-effect cone-correction as per Gavara et al. ${ }^{53}$. Data for force spectroscopic curves of AFM tip displacement against indentation force were rejected when discontinuities in the curves were present, corresponding to samples slipping and an inaccurate indentation. For the sample shown in Fig. 1d-g, only two independent experimental replicates were fabricated, as this sample only served to illustrate how a chemomechanically voxelated niche material could be fabricated with the CMFL methodology. One replicate was mounted for confocal microscopy (Fig. 1d, e), and the other was analyzed with force spectroscopy (Fig. 1e, f). Microstructured linewidth and bioconjugation were measured using confocal microscopy. Linewidth was directly measure using Fiji-ImageJ across three independent experimental replicates

360 with quantification of the concentration of Biotin-PEG-SH was measured indirectly by measuring the relative fluorescence of bound streptavidin-FITC. Indirect measurement was used to prevent the photobleaching or free radical attack of fluorescent molecules during photopolymerization, that otherwise limited interpretation.

Interpolation method for voxelated niches microproperties. Empirical data was tabulated 365 pairing dependent and independent variables, including Young's modulus, linewidth, and the concentration of bioconjugate (Fig. 2g-i), with the monomer-photoinitiator ("PEG/PI" below), focus (shown as " $Z$ ") and [Biotin-PEG-SH]. Using MATLAB (2020a), we then calculated the value of the independent fabrication variables of $P E G / P I$ and $Z$ after substitution of the desired Young's modulus $\left(E_{\text {des. }}\right)$ and linewidth $\left(W_{\text {des. }}\right)$ as per Equation 1 below:

$$
0=\left(\frac{E_{\text {des. }} . \frac{\sum\left(\tau * E_{\text {em }}\right)}{\Sigma \tau}}{E_{\text {des. }}}\right)^{2}+\left(\frac{W_{\text {des. }}-\frac{\sum(\tau * W \text { em. })}{\Sigma \tau}}{W_{\text {des. }}}\right)^{2}, \text { where } \tau=\frac{1}{0.1 \sqrt{2 \pi}} e^{-\frac{1}{2}\left(\frac{\left|\frac{P E G / P I_{\text {em. }} . P E G / P I}{40}\right|+\left|\frac{Z_{\text {em. }} .}{6}\right|}{0.1}\right)} \text { Equ. }
$$

where, $P E G / P I_{e m}$ and $Z_{e m}$ are vectors from the independent paired variables from the tabulated empirical dataset presented in Fig. 2g,h, and are used to calculate the vector $\tau$ that is substituted into Equ. 1, where a solution is obtained for the dependent paired variables $E_{e m}, W_{e m}$. The numbers 40 and 6 in Equ. 1 represent the normalization range for $P E G / P I$ and $Z$ respectively, over which data is interpolated. Equ. 1 is solved using the Nelder-Mead simplex method for finding the 
minimum of unconstrained multivariable functions. The relationship between $P E G / P I$ is calculated as the positive real solution of Equ 2.

$$
0=\left|\left(\frac{P E G / P I-50}{40}\right)^{2}+\left(\frac{P I-0.5}{0.35}\right)^{2}\right|-1 \text { Equ. }
$$

380 Cell culture. hADSCs (Life Technology) were cultured in expansion media: MesenPRO RSTM Basal Medium (Invitrogen) with the supplement of $2 \mathrm{mM}$ l-glutamine and MesenPRO RS Growth Supplement (Life Technology). hADSCs at passage 3 were used for all the studies. For culture on niches, a $10 \mathrm{~mm}$ coverslip with printed niche arrays was placed in a 48 -well plate. Niche arrays were washed twice with PBS before 12 mins UV-light sterilization using a biosafety cabinet. The medium was changed every 3 days except for studies examining YAP and RUNX2 nuclear translocation, where hADSCs were treated with mitomycin $\left(10 \mu \mathrm{g} \mathrm{mL}{ }^{-1}\right)$ (Cayman Chemical, 11435 ) for $2 \mathrm{~h}$, to inhibit proliferation, $24 \mathrm{~h}$ after seeding, after which media was replaced with expansion media. For YAP and RUNX2 translocation studies, cells were seeded at 3,000 cells cm

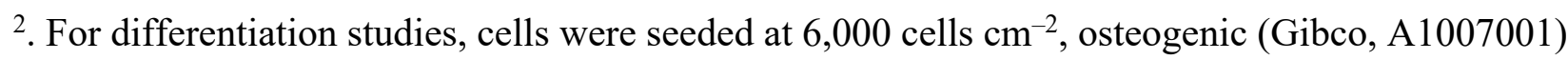
390 and adipogenic media (Gibco, A1007201) were used as noted. Experiments using iPSCs were performed with the CRL2429 C16 hiPSC line, which was derived at the Australian Institute for Bioengineering and Nanotechnology (AIBN) at the University of Queensland as previously described $^{54}$. For routine passaging, cells were passaged with ReLeSR ${ }^{\mathrm{TM}}$ and grown on Stem Cell Grade Matrigel ${ }^{\circledR}$ (Corning) coated 6-well plate. All experiments used mTeSR ${ }^{\mathrm{TM}}$ plus, with supplements as listed. For cell culture of iPSCs on niches, a $10 \mathrm{~mm}$ coverslip with printed niche arrays was placed in a 48-well plate. The niche arrays were washed twice with PBS before 12 mins UV-sterilization using a biosafety cabinet. C16 hiPSC cells were dissociated with Accutase (Gibco) and pipetted into a single cell suspension in $\mathrm{mTeSR}^{\mathrm{TM}}$ plus supplemented with $10 \mu \mathrm{M}$ Rockinhibitor Y27632 (Ri) at 3.3M cells $\mathrm{mL}^{-1}$ before seeding at $300 \mu \mathrm{L}$ per well. After $2 \mathrm{~h}$, media was 400 replaced with $10 \mu \mathrm{M}$ Ri for $10 \mathrm{~h}$, before $48 \mathrm{~h}$ differentiation in $500 \mu \mathrm{L}$ mTeSR ${ }^{\mathrm{TM}}$ plus supplemented with $50 \mathrm{ng} \mathrm{mL} \mathrm{m}^{-1}$ BMP4 (PHC9534 Gibco). For all cell culture experiments, a minimum of 3 independent experimental replicates was completed for each printed fabrication condition and relevant stain. All cells tested negative for mycoplasma contamination. For hADSCs, routine PCR assay checks (LookOut ${ }^{\circledR}$ Mycoplasma qPCR Detection Kit, MP0040A-1KT) were completed for 405 mycoplasma contamination, for hiPSC mycoplasma was tested using the fluorescent kit, MycoAlertTM Mycoplasma Detection Kit, Catalog \#: LT07-318.

Immunofluorescence, confocal imaging, and image quantification. For immunostaining, all solutions, except for those with dilute antibody and fluorophores, were syringe filtered through $0.22 \mu \mathrm{m}$ membrane filters (Merck Millipore SLGP033RS). Cells were fixed at room temperature 
$4104 \%$ PFA in $\mathrm{x} 1$ PBS buffer for 10 mins and then washed three times with PBS, followed by $12 \mathrm{~min}$ permeabilization at room temperature with $0.1 \mathrm{w} \mathrm{v}^{-1} \%$ Triton X-100 in PBS. Samples were then incubated in a blocking buffer of 3\% BSA, $3.75 \mathrm{mg} \mathrm{mL}^{-1}$ glycine, and $0.05 \% \mathrm{w} \mathrm{v}^{-1}$ Tween 20 in PBS for $1 \mathrm{~h}$ at room temperature. Primary antibodies were diluted in $1 \%$ BSA and $0.05 \% \mathrm{w} \mathrm{v}^{-1}$ Tween 20 in PBS and added overnight at $4^{\circ} \mathrm{C}$ (anti-YAP (1:250; Santa Cruz sc-101199), anti-

415 RUNX2-AF488 (1:250; Santa Cruz sc-390351)), or for 2h ( (1:400) SOX2, CST \#3579; (1:300) SOX17, R\&D systems, AF1924; (1:300) BRA, R\&D Systems, AF2085). For indirect immunostaining, samples were washed $\mathrm{x} 3$ times with PBS and incubated for $2 \mathrm{~h}$ at room temperature with corresponding secondary antibody (YAP with Santa Cruz CFL 488 (1:250, sc516176), SOX2 with ab150075, SOX17, and BRA with ab150077). Nuclear and actin counterstains 420 were performed using Abcam iFluor conjugated phalloidin (ab176753, ab176759), and Hoechst 33342 at $0.1 \mu \mathrm{g} \mathrm{mL}-1$ (Sigma, 14533) dilute in x1 PBS and incubated for $30 \mathrm{mins}$ at room temperature. Following counterstain incubation, samples were washed an additional $\mathrm{x} 3$ with PBS containing $0.05 \% \mathrm{w} \mathrm{v}^{-1} \%$ sodium azide. All microstructured niche samples were mounted in PMMA blocks with a recessed circular hole to avoid damaging samples (Fig. S9). For YAP and 425 RUNX2 translocation studies, microscopy was completed on a Zeiss LSM 800 Confocal microscope using 63x Objective Plan-Apochromat 63x/1.40NA Oil objective (with an in-plane lateral resolution of $0.413-0.124 \mu \mathrm{m}$ per pixel) and pinhole diameter of $1.0 \mathrm{AU}(50.34 \mu \mathrm{m})$ and azimuthal resolution of $0.4 \mu \mathrm{m}$. Nuclear images from Hoechst staining were used to creates masks that define a nuclear volume. Cytoplasmic masks were defined from flood-filled phalloidin stains,

430 with the average fluorescent intensity of each volume calculated in MATLAB Therein, the YAP/RUNX2 nuclear to cytoplasmic translocation ratio was determined as the ratio of the mean YAP/RUNX2 fluorescent saturation intensity of the nuclear volume divided by the fluorescent saturation intensity in the non-nuclear cell cytoplasmic volume. In Fig. 3b,c, 4a,c, YAP/RUNX2 measurements of a total of at least 12 single cells per condition were pooled across 3 independent 435 experimental replicates from at least 4 printed-niche-replicates. Representative images were selected according to their proximity to the mean data as calculated across all replicates. For imaging hiPSCs over $1 \mathrm{~mm}$ square microstructures, we used a 10x Nikon A1R confocal microscope with an in-plane lateral resolution of $0.615 \mu \mathrm{m}$ per pixel (2048x2048 pixels) and an azimuthal resolution of 2.5-3.3 $\mu \mathrm{m}$. Using Hoechst nuclear marker, individual cell nuclei were segmented 440 using Trainable Weka Segmentation, defining three different pixel classes, including nuclearcontours, nuclei, and background. Probability maps were generated and used to identify individual cell nuclei with a loss function that assigns pixels to the final nuclei mask given they: have at least $50 \%$ chance of being nuclei and at most a $66 \%$ and $75 \%$ chance of being a pixel belonging to the 17 
nuclear-contour and background pixel-classes respectively. Using the nuclei masks, the mean

445 fluorescent intensity of each channel in each nucleus was calculated. Then the coordinates of each nucleus within the niches were calculated, allowing replicate data to be remapped to a single plot that showed the average position mapped immunostained expression of the 3 germ layer channels with marker-size and marker-opacity changing proportionally to the fluorescent intensity of the nuclei (see SI for additional details). The expression maps in Fig. 5b,c pool data from 3 independent experimental replicates, analyzing at least 15 printed-niche-replicates and at least 101,594 individual cells for each marker.

LipidTOX, CNA35, and Alizarin Red staining of ADSC differentiation. We assayed the differentiation of hADSCs toward adipogenic and osteogenic lineage in response to niche microproperties. All solutions listed below were syringe filtered through $0.22 \mu \mathrm{m}$ membrane filters

455 (Merck Millipore SLGP033RS). Alizarin Red (Sigma, A5533) staining was performed to examine the presence of mineralized deposits under osteogenic differentiation conditions. Samples were washed $\mathrm{x} 2$ with PBS before fixation at room temperature in 4\% PFA dilute in $\mathrm{x} 1$ PBS buffer for 10 mins and then washed three times with PBS. Samples were then washed x3 in Milli-Q H2O before incubation with Alizarin Red stain for 5 minutes $(9.6 \mathrm{mg} \mathrm{mL}-1$ Alizarin Red at a $\mathrm{pH}$ of 4.2, 460 adjusted with acetic acid). Following incubation, samples were washed x5 with Milli-Q H2O, followed by a further $\mathrm{x} 3$ washes with $\mathrm{x} 1 \mathrm{PBS}$ containing $0.05 \mathrm{w} \mathrm{v}-1 \%$ sodium azide. We examined cell mineralization with Alizarin Red staining and widefield Colour microscopy of materials following $14 \mathrm{~d}$ of culture. Cells were imaged using a Nikon Ni E microscope with color DS-Fi2 camera and Plan Apo Lambda 10x/0.45NA dry objective. The localization of osteogenesis over

465 specified RGD and Young's modulus regions was quantified for each niche-replicate sample. The mean red saturation was divided by the mean total saturation that combines red, green, and blue color components for the corresponding region of interest. For Alizarin Red Stains in Fig. 4 b,d,h,k, measurements were pooled across 3 independent experimental replicates from at least 4 printedniche-replicates in each experiment for a total of at least 12 total measurements per niche condition.

470 Staining with LipidTOX Red Neutral Lipid Stain (Thermo H34476) was completed to quantify cell fat volume under adipogenic differentiation conditions. Samples were fixed at room temperature 4\% PFA in $\mathrm{x} 1 \mathrm{PBS}$ buffer at $\mathrm{pH} 7.4$ for 10 mins and then washed three times with PBS, followed by 12 min permeabilization at room temperature with $0.1 \mathrm{w} \mathrm{v}-1 \%$ Triton X-100 in PBS. Samples were then incubated with LipidTOX Red Neutral Lipid Stain (diluted 1:800), Abcam iFluor 475 conjugated phalloidin (1:200), and Hoechst 33342 at $0.1 \mu \mathrm{g} \mathrm{mL}-1$ (Sigma, 14533) dilute in x1 PBS for 30 mins at room temperature. For LipidTOX data in Fig. 4 e,f, measurements were pooled across 3 independent experimental replicates from at least 4 printed-niche-replicates in each 18 
experiment for a total of at least 24 fields-of-view per niche condition. In Fig. 4 k, LipidTOX measurements were pooled across 3 independent experimental replicates from at least 4 printedniche-replicates in each experiment for a total of 12 organotypic bone-fat niches. Following incubation, samples were washed an additional $\mathrm{x} 3$ with $\mathrm{x} 1$ PBS containing $0.05 \mathrm{w} \mathrm{v}-1 \%$ sodium azide. Fluorescent microscopy of large fields of view (arrays of RGD and Young's modulus) were tiled using a Nikon Ni E microscope with a motorized stage and monochrome DS-Qi2 camera and Plan Apo Lambda 10x/0.45NA dry objective. Confocal microscopy was completed on a Zeiss LSM 800 Confocal microscope using x63 Objective Plan-Apochromat 63x/1.40NA Oil objective (with an in-plane lateral resolution of $0.413 \mu \mathrm{m}$ per pixel), pinhole diameter of $1.0 \mathrm{AU}(50.34 \mu \mathrm{m})$, and azimuthal resolution of $0.4 \mu \mathrm{m}$. Segmentation was performed using custom MATLAB scripting that makes use of the open microscopy Bio-Formats tool. Masks were created to define cytoplasmic and fat volumes using phalloidin and LipidTOX stains. For production and purification of the 490 fluorescent collagen 1A probe, the pET28a-EGFP-CNA35 plasmid was received as a gift from Maarten Merkx (Addgene plasmid \# 61603; http://n2t.net/addgene:61603; RRID: Addgene_61603) and synthesized as reported previously ${ }^{55}$. In brief, protein yields of the CNA35 probe were synthesized using E.Coli bacteria before purification using ÄKTApurifier (Cytiva) and a $5 \mathrm{ml} \mathrm{Ni-NTA} \mathrm{Superflow} \mathrm{Cartridge} \mathrm{(Qiagen),} \mathrm{dialysis} \mathrm{with} \mathrm{SnakeSkinTM} \mathrm{Dialysis} \mathrm{tubing} \mathrm{with} 10$ $495 \mathrm{kDa} \mathrm{MWCO}$, and concentration with an Amicon $10 \mathrm{kDa} \mathrm{MWCO}$ centrifugal filter unit. For imaging, $0.5 \mu \mathrm{M}$ of EGFP-CNA35 solution were added to the sample and incubated on a plate rocker for 15 mins, before washing twice with PBS. In Fig. 4 k, Col1A measurements were pooled across 3 independent experimental replicates from at least 4 printed-niche-replicates in each experiment for a total of 12 organotypic bone-fat niches.

Acknowledgments. The authors gratefully acknowledge the Australian Government's financial support for providing PLHN an Australian Postgraduate Award Scholarship, the Australian Department of Education and Training for awarding PLHN with an Endeavour Fellowship. A massive thank you to the discussions and support of Dr. Martin Stewart, Dr. Christina Viray, Dr.

505 Ashnil Kumar, and Hélène Lebhar from the UNSW Recombinant Products Facility for assistance in the production and purification of EGFP-CNA35. From Dr. Courtney Wright as the mensch, and the facilities and the scientific and technical aid of Microscopy Australia at the Australian Centre for Microscopy \& Microanalysis at the University of Sydney, including Dr. Ying Ying Su and Dr. Neftali Flores-Rodriguez. PN thanks Karl Gifford for his assistance with the custom Hyrel System $30 \mathrm{M}$ 's modification and operation. 
Author Contributions. PN conceived of the work, designed the modified CMFL 3D-printer device and method, designed and executed all experimentation, analyzed all data, and wrote the manuscript. PO assisted manuscript preparation, and together with JS, helped the development of

515 methods relating to hiPSCs. DK prepared and provided CNA fluorescent probes and methods relating to their use. MB provided scientific oversight, experimental analysis and assisted with manuscript preparation. PT funded experiments relating to hiPSCs, assisted experimental design and methods on the culture of hiPSCs, provided scientific oversight, and assisted with manuscript preparation. J-WS helped to optimize the methods for the specification of niche mechanics and

520 force spectroscopic methods. HZ funded the project, provided scientific oversight, and assisted with manuscript preparation.

Sources of research support. Australian Postgraduate Award Scholarship, Australian Endeavour Award, and Cardiovascular Institute ECR grant (to PLHN.), The Australian National Health and 525 Medical Research Council, National Institutes of Health Grant No. R00-HL125884 (to J-WS). APP1107470 NHMRC Senior Research Fellowship and APP1139515 NHMRC Project grant, and Australian Research Council ITTC IC170100022 (to HZ.)

Competing Interests statement. The authors declare no competing interests.

Code availability statement. No standalone software or software tools were developed for this work. However, all scripts used for data analysis are available upon request to the corresponding authors.

535 Data availability statement. Datasets supporting the conclusions are shown within the article and its additional files. Data and related scripts with sample data for analysis are available upon request to the corresponding authors.

\section{References.}

540 1. Warmflash, A., Sorre, B., Etoc, F., Siggia, E. D. \& Brivanlou, A. H. A method to recapitulate early embryonic spatial patterning in human embryonic stem cells. Nat. Methods 11, 847 (2014).

2. Nikolaev, M. et al. Homeostatic mini-intestines through scaffold-guided organoid morphogenesis. Nature (2020) doi:10.1038/s41586-020-2724-8. 
3. Brassard, J. A., Nikolaev, M., Hübscher, T., Hofer, M. \& Lutolf, M. P. Recapitulating macroscale tissue self-organization through organoid bioprinting. Nat. Mater. (2020) doi:10.1038/s41563-020-00803-5.

4. Manfrin, A. et al. Engineered signaling centers for the spatially controlled patterning of human pluripotent stem cells. Nat. Methods 16, 640 (2019).

5. Zheng, Y. et al. Controlled modelling of human epiblast and amnion development using stem 550 cells. Nature 573, 421-425 (2019).

6. Birey, F. et al. Assembly of functionally integrated human forebrain spheroids. Nature 545, 54 59 (2017).

7. Engler, A. J., Sen, S., Sweeney, H. L. \& Discher, D. E. Matrix elasticity directs stem cell lineage specification. Cell 126, 677-689 (2006).

555 8. Cameron, A. R., Frith, J. E. \& Cooper-White, J. J. The influence of substrate creep on mesenchymal stem cell behaviour and phenotype. Biomaterials 32, 5979-5993 (2011).

9. Dalby, M. J., Gadegaard, N. \& Oreffo, R. O. Harnessing nanotopography and integrin-matrix interactions to influence stem cell fate. Nat. Mater. 13, 558-569 (2014).

10. Khetan, S. et al. Degradation-mediated cellular traction directs stem cell fate in covalently 560 crosslinked three-dimensional hydrogels. Nat. Mater. 12, 458 (2013).

11. Hosaka, S., Ozawa, H. \& Tanzawa, H. Controlled release of drugs from hydrogel matrices. J. Appl. Polym. Sci. 23, 2089-2098 (1979).

12. Edelman, E. R., Mathiowitz, E., Langer, R. \& Klagsbrun, M. Controlled and modulated release of basic fibroblast growth factor. Biomaterials 12, 619-626 (1991).

565 13. Burdick, J. A. \& Anseth, K. S. Photoencapsulation of osteoblasts in injectable RGD-modified PEG hydrogels for bone tissue engineering. Biomaterials 23, 4315-4323 (2002).

14. Cosgrove, B. D. et al. N-cadherin adhesive interactions modulate matrix mechanosensing and fate commitment of mesenchymal stem cells. Nat. Mater. 15, 1297 (2016).

15. Miller, J. S. et al. Rapid casting of patterned vascular networks for perfusable engineered

570 three-dimensional tissues. Nat. Mater. 11, 768-774 (2012).

16. Xu, T. et al. Complex heterogeneous tissue constructs containing multiple cell types prepared by inkjet printing technology. Biomaterials 34, 130-139 (2013).

17. Hockaday, L. et al. Rapid 3D printing of anatomically accurate and mechanically heterogeneous aortic valve hydrogel scaffolds. Biofabrication 4, 035005 (2012).

575 18. Kolesky, D. B., Homan, K. A., Skylar-Scott, M. A. \& Lewis, J. A. Three-dimensional bioprinting of thick vascularized tissues. Proc. Natl. Acad. Sci. 113, 3179-3184 (2016). 
19. Kang, H.-W. et al. A 3D bioprinting system to produce human-scale tissue constructs with structural integrity. Nat. Biotechnol. 34, 312-319 (2016).

20. Ober, T. J., Foresti, D. \& Lewis, J. A. Active mixing of complex fluids at the microscale. Proc. Natl. Acad. Sci. 112, 12293-12298 (2015).

21. Skylar-Scott, M. A., Mueller, J., Visser, C. W. \& Lewis, J. A. Voxelated soft matter via multimaterial multinozzle 3D printing. Nature 575, 330-335 (2019).

22. Lee, A. et al. 3D bioprinting of collagen to rebuild components of the human heart. Science 365, 482 (2019).

585 23. Kolesky, D. B. et al. 3D bioprinting of vascularized, heterogeneous cell-laden tissue constructs. Adv. Mater. 26, 3124-3130 (2014).

24. Richter, B. et al. Guiding cell attachment in 3D microscaffolds selectively functionalized with two distinct adhesion proteins. Adv. Mater. 29, 1604342 (2017).

25. Ma, X. et al. Deterministically patterned biomimetic human iPSC-derived hepatic model via 590 rapid 3D bioprinting. Proc. Natl. Acad. Sci. 113, 2206-2211 (2016).

26. Lawlor, K. T. et al. Cellular extrusion bioprinting improves kidney organoid reproducibility and conformation. Nat. Mater. 1-12 (2020).

27. Müller, P. et al. STED-Inspired Laser Lithography Based on Photoswitchable Spirothiopyran Moieties. Chem. Mater. 31, 1966-1972 (2019).

595 28. Chen, C. S., Mrksich, M., Huang, S., Whitesides, G. M. \& Ingber, D. E. Geometric control of cell life and death. Science 276, 1425-1428 (1997).

29. Mosiewicz, K. A. et al. In situ cell manipulation through enzymatic hydrogel photopatterning. Nat. Mater. 12, 1072 (2013).

30. Luo, Y. \& Shoichet, M. S. A photolabile hydrogel for guided three-dimensional cell growth 600 and migration. Nat. Mater. 3, 249 (2004).

31. Shadish, J. A., Benuska, G. M. \& DeForest, C. A. Bioactive site-specifically modified proteins for 4D patterning of gel biomaterials. Nat. Mater. 1 (2019).

32. Yin, H., Ding, Y., Zhai, Y., Tan, W. \& Yin, X. Orthogonal programming of heterogeneous micro-mechano-environments and geometries in three-dimensional bio-stereolithography. Nat. 605 Commun. 9, $4096(2018)$.

33. Mayer, F. et al. Multimaterial 3D laser microprinting using an integrated microfluidic system. Sci. $A d v$. 5, eaau9160 (2019).

34. Dendukuri, D., Pregibon, D. C., Collins, J., Hatton, T. A. \& Doyle, P. S. Continuous-flow lithography for high-throughput microparticle synthesis. Nat. Mater. 5, 365 (2006). 
610 35. Fairbanks, B. D., Schwartz, M. P., Bowman, C. N. \& Anseth, K. S. Photoinitiated polymerization of PEG-diacrylate with lithium phenyl-2, 4, 6-trimethylbenzoylphosphinate: polymerization rate and cytocompatibility. Biomaterials 30, 6702-6707 (2009).

36. Guimarães, C. F., Gasperini, L., Marques, A. P. \& Reis, R. L. The stiffness of living tissues and its implications for tissue engineering. Nat. Rev. Mater. 1-20 (2020).

615 37. Dupont, S. et al. Role of YAP/TAZ in mechanotransduction. Nature 474, 179-183 (2011).

38. Yang, C., Tibbitt, M. W., Basta, L. \& Anseth, K. S. Mechanical memory and dosing influence stem cell fate. Nat. Mater. 13, 645-652 (2014).

39. Caliari, S. R., Vega, S. L., Kwon, M., Soulas, E. M. \& Burdick, J. A. Dimensionality and spreading influence MSC YAP/TAZ signaling in hydrogel environments. Biomaterials 103, 314 $620323(2016)$.

40. Meng, Z., Moroishi, T. \& Guan, K.-L. Mechanisms of Hippo pathway regulation. Genes Dev. 30, 1-17 (2016).

41. Chaudhuri, O. et al. Hydrogels with tunable stress relaxation regulate stem cell fate and activity. Nat. Mater. 15, 326-334 (2016).

625 42. Komori, T. Regulation of bone development and extracellular matrix protein genes by RUNX2. Cell Tissue Res. 339, 189 (2010).

43. Muncie, J. M., Ayad, N. M., Lakins, J. N. \& Weaver, V. M. Mechanics regulate human embryonic stem cell self-organization to specify mesoderm. Dev.-CELL--20-00131 (2020).

44. Tewary, M. et al. A stepwise model of reaction-diffusion and positional information governs 630 self-organized human peri-gastrulation-like patterning. Development 144, 4298-4312 (2017).

45. Xue, X. et al. Mechanics-guided embryonic patterning of neuroectoderm tissue from human pluripotent stem cells. Nat. Mater. 17, 633-641 (2018).

46. Fu, J., Warmflash, A. \& Lutolf, M. P. Stem-cell-based embryo models for fundamental research and translation. Nat. Mater. 1-13 (2020).

635 47. Nakaya, Y., Sukowati, E. W., Wu, Y. \& Sheng, G. RhoA and microtubule dynamics control cell-basement membrane interaction in EMT during gastrulation. Nat. Cell Biol. 10, 765-775 (2008).

48. Kyprianou, C. et al. Basement membrane remodelling regulates mouse embryogenesis. Nature 1-6 (2020).

640 49. Martyn, I., Kanno, T., Ruzo, A., Siggia, E. \& Brivanlou, A. Self-organization of a human organizer by combined Wnt and Nodal signalling. Nature 558, 132-135 (2018).

50. Grigoryan, B. et al. Multivascular networks and functional intravascular topologies within biocompatible hydrogels. Science 364, 458-464 (2019). 
51. Wylie, R. G. et al. Spatially controlled simultaneous patterning of multiple growth factors in 645 three-dimensional hydrogels. Nat. Mater. 10, 799 (2011).

52. Gjorevski, N. et al. Designer matrices for intestinal stem cell and organoid culture. Nature 539, 560-564 (2016).

53. Gavara, N. \& Chadwick, R. S. Determination of the elastic moduli of thin samples and adherent cells using conical atomic force microscope tips. Nat. Nanotechnol. 7, 733 (2012).

650 54. Briggs, J. A. et al. Integration-free induced pluripotent stem cells model genetic and neural developmental features of down syndrome etiology. Stem Cells 31, 467-478 (2013).

55. Aper, S. J. et al. Colorful protein-based fluorescent probes for collagen imaging. PloS One 9, e114983 (2014). 